\title{
On the Quantum Theory of Radiation
}

\author{
By \\ Tareq Ahmed Mokhiemer \\ Research Assistant, Physics Department \\ KFUPM
}

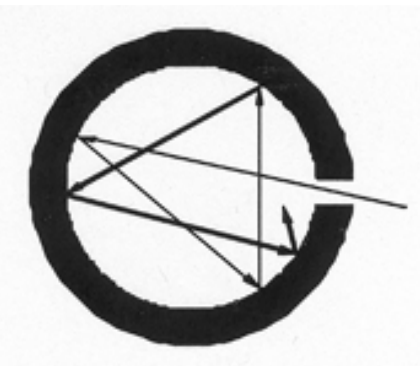

Abstract

The Planck's law of blackbody radiation is derived in several methods in this paper. The original Planck's derivation in his 1900 paper, the derivation by Einstein, and another derivation based on the Bose-Einstein statistics are reviewed with comments on each derivation. Statistical properties of radiation are briefly reviewed in the second part of the paper. Different concepts of photon statistics are applied on the thermal (chaotic) light and laser light. Nonclassical properties of light that manifest its quantum nature are briefly highlighted with a short review of the experimental evidence embodied in 


\section{1-Introduction and historical perspective}

It was Robert Kirchhoff who first argued in 1859 that the thermal radiation of the blackbody was of a fundamental nature. ${ }^{1}$ Since that time, several attempts were made to derive a precise relation that matches the experimental results. Wien, suggested that the radiation density per unit frequency per unit volume depended on frequency and temperature by the relation $\mathrm{u}(\mathrm{f}, \mathrm{T})$ $=\mathrm{af}^{3} \exp (\mathrm{bf} / \mathrm{T})$. This law corresponded well with the experiment but had no theoretical foundation ${ }^{1}$. Rayleigh and James Jeans analyzed the problem from a classical point of view and they came up with a relation that was drastically different than experiment (the ultraviolet catastrophe). This contradiction was an indication of some problems in classical physics. It was Max Planck who first reached a sound proof of the radiation density formula based on the statistical definition of the entropy and its relation to the energy and temperature. Planck had to assume that the energies of the harmonic oscillators that emit the radiation are quantized to arrive at the correct relation that describes the experimental observations at short and long wavelengths.

Einstein's work on the photoelectric effect and the specific heat of metals, has drawn the attention to the role that the theory of quanta may play in physics. As a response to these radical developments in physics, a conference was held in Solvay in 1911, on "radiation theory and the quanta" to discuss the emerging quantum theory. Henri Poincare was one of the attendants of this conference. Although he spent most of his life as a classical physicist and a prominent mathematician, he became excited by the idea of quantization. Unsatisfied by Planck's proof of the blackbody radiation formula, he worked on his own proof. In his last memoir, written six months before his death, Poincare tried to prove that not only quantization was a sufficient condition for deriving the blackbody radiation formula, but also a necessary condition. The blackbody radiation formula remains today as important as it was in the beginning of the twentieth century, since it has opened the door for 'further quantization' and the beginning of the quantum mechanics.

\section{2-Planck's derivation}

In his famous paper, Planck used the model of harmonic oscillators for the frequencies of the radiation. His motivation to use the model of a harmonic oscillator may be to model the dipole radiation where charge oscillation leads to the emission of electromagnetic waves. It was known that such Hertzian resonators by virtue of their vibration, radiate hertzian( Electromagnetic) waves. ${ }^{7}$ The simplest mechanical model that has oscillation is the harmonic oscillator. These oscillators were thought to exist in the walls of the cavity (what we now know to be atoms). It's not clear to me however why Planck opted to use $\mathrm{N}$ harmonic 
oscillator for each frequency and not a single one. Planck started by stating cleverly that a single harmonic oscillator with constant phase and amplitude has zero entropy since it's in complete order (and entropy is a measure of the amount of disorder). In his own words, Planck said: "Entropy depends on disorder and this disorder, according to the electromagnetic theory of radiation for the monochromatic vibrations of a resonator when situated in a permanent stationary radiation field, depends on the irregularity with which it constantly changes its amplitude and phase, provided one considers time intervals large compared to the time of one vibration but small compared to the duration of a measurement. If amplitude and phase both remained absolutely constant, which means completely homogeneous vibrations, no entropy could exist and the vibrational energy would have to be completely free to be converted into work." However as we will see later at the end of his proof, the single harmonic oscillator turned out to have a nonzero entropy. To prove his famous relation, Planck had to quantize the energy of the oscillators and consider many harmonic oscillators instead of only one and calculate the entropy by counting the number of ways by which the total energy can be distributed among these oscillators( the number of possible microstates).

By assuming that the energy quantum $=\varepsilon$ and the total energy $\mathrm{UN}=\mathrm{P} \varepsilon$, the number of ways to distribute $\mathrm{P}$ quanta among $\mathrm{N}$ distinguishable oscillators is given by:

$$
R=\frac{(N+P-1) !}{P !(N-1) !}
$$

Then the enropy directly follows from Boltzmann's law as $\mathrm{SN}=\mathrm{k} \log \mathrm{R}$

$\mathrm{SN}=\mathrm{k}\{(\mathrm{N}+\mathrm{P}) \log (\mathrm{N}+\mathrm{P})-\mathrm{N} \log \mathrm{N}-\mathrm{P} \log \mathrm{P}\}$ and in terms of $\mathrm{U}$, e the entropy can be written as:

$$
S^{N}=k N\left\{\left(1+\frac{U}{\epsilon}\right) \log \left(1+\frac{U}{\epsilon}\right)-\frac{U}{\epsilon} \log \frac{U}{\epsilon}\right\}
$$

And the entropy of a single harmonic oscillator is given by the previous quantity divided by $\mathrm{N}$, namely:

(6) $S=k\left\{\left(1+\frac{U}{\epsilon}\right) \log \left(1+\frac{U}{\epsilon}\right)-\frac{U}{\epsilon} \log \frac{U}{\epsilon}\right\}$

How did Planck explain the source of the entropy (disorder) in the single oscillator, in contrast to what he mentioned in the beginning of his article? If he was asked that question after 1927, he may have said it is due to the uncertainty relation between the two conjugate 
variable P, Q in the harmonic oscillator, but what if he was asked in 1900 ?? How could he reach the same result if he starte by a single harmonic oscillator (i.e, $\mathrm{N}=1$ ) ??

A direct application of the relation between Temperature, entropy, and Energy of a single oscillator $\frac{1}{\theta}=\frac{d S}{d U}$ to the previous equation yields directly $U=\frac{\varepsilon}{e^{\frac{\varepsilon}{k \theta}}-1}$. Then as Einstein did, Planck made use of Wien's law to derive the specific relation between the quantum $\varepsilon$ and the frequency of the oscillator $v$. Wien's law implies that the relation between the energy density $u$, and the frequency and temperatures is given by:

$$
\mathrm{u}=\frac{v^{3}}{\mathrm{c}^{3}} \cdot f\left(\frac{\theta}{v}\right)
$$

Using the relation between the radiation density and the energy of the single oscillator that Planck derived in an earlier paper $u=\frac{8 \pi v^{2}}{c^{3}} U$, he could rewrite Wien's law in the form

$$
\mathrm{U}=v \cdot f\left(\frac{\theta}{v}\right)
$$

By comparing this relation with the expression for $U$ derived earlier, it's seen that the energy of the smallest quantum is given by $\varepsilon=h v$, where $\mathrm{h}$ is a universal constant.

Combining the previous result and the expression of the radiation density which is the energy per unit volume per unit frequency interval we obtain the famous Planck law

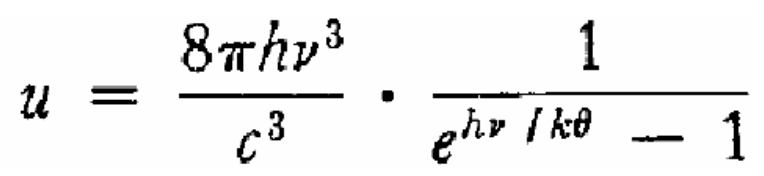

In the time Planck intrduced this assumption of the quantization of the energy of a harmonic oscillator, nobody noticed how profound this assumption was in the physics. I don't why didn't Planck continue in the same line of thought and ask himself immediately how or why was the energy of the harmonic oscillator quantized? It was not before 25 years later that the real reason of quantizing the energy of the harmonic oscillator was rigoursly derived from the postulates of quantum mechanics.

\section{3- Einstein derivation of Planck's radiation law}

Although the famous paper in which Einstein introduced the A, B coefficients was written mainly to derive the Planck's radiation law for the blackbody problem, this derivation is usually forgotten and the paper is remembered only for laying the foundations for the operation of the laser. The derivation goes as follows:

Einstein assumed that the atoms of the walls of the blackbody are two-level atoms where they can absorb or emit quanta of energy equal the same amount as the difference between the two levels. Each atom has an absorption and emission probability $\mathrm{Pa}, \mathrm{Pe}$ given by 


$$
\begin{aligned}
& p_{a}=B_{a} \rho_{\nu}, \\
& p_{e}=A+B_{e} \rho_{\nu},
\end{aligned}
$$

Where $\rho_{v}$ is the density of electromagnetic radiation in the cavity, $\mathrm{Ba}$ and $\mathrm{Be}$ are undetermined coefficients for absorption and stimulated emission, respectively, and $\mathrm{A}$ is the coefficient for spontaneous emission. ${ }^{\text {a }}$ Let the number of atoms per unit volume in the excited state and ground state be $n_{e}, n_{g}$ respectively. The detailed balance condition which results from the thermal equilibrium between the radiation and the atoms states that the probability that an atom in the ground state gets excited equals the probability that an excited atom emits the photon and decays to the ground state. This condition can be expressed as:

$$
p_{a} n_{g}=p_{e} n_{e}
$$

Then Einstein assumed that the atoms themselves are in thermal equilibrium ${ }^{b}$ and he used the partition function derived from the canonical ensemble to write the ratio between $n_{e}$ and $\mathrm{n}_{\mathrm{g}}$ as

$$
\frac{n_{e}}{n_{g}}=e^{-\epsilon / k T}
$$

Solving the four previous equations for $\rho_{v}$ we find that

$$
\rho_{\nu}=\frac{A}{\left(B_{a} e^{\epsilon / k T}-B_{e}\right)}
$$

Which is indeed similar to Planck's formula. Next, Einstein made use of the results reached by Rayleigh about the relation between the radiation density and the frequency at long wavelengths and high temperatures namely

$$
\rho_{\nu}=\gamma \nu^{2} k T
$$

This limit can be indeed satisfied if we choose $\mathrm{Ba}=\mathrm{Be}=\mathrm{B}$ where the radiation density will be

in the form $\rho_{v}=A / B \frac{\varepsilon}{k T}$. To determine the dependence of $\mathrm{A} / \mathrm{B}$ and $\varepsilon$ on frequency,

Einstein used another law that states that $\rho_{v}$ has a scaling dependence on the variables $v$ and T, (Wien's displacement law) of the form

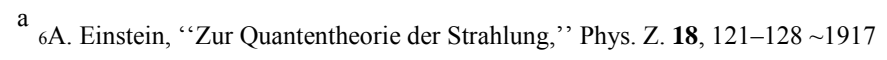

${ }^{b}$ The atoms of course are continuously absorbing and emitting photons, but since they are in equilibrium with the radiation, their total energy is fixed and hence they can be considered as an isolated ensemble. 


$$
\rho_{\nu}=\nu^{3} f(\nu / T)
$$

To satisfy this equation the only choices for $\mathrm{A} / \mathrm{B}$ and $\varepsilon$ will be

$$
\begin{aligned}
& \epsilon=h \nu, \\
& \frac{A}{B}=\alpha \nu^{3}
\end{aligned}
$$

Where h, $\alpha$ are universal constants. Finally the energy density of the radiation will look like

$$
\rho_{v}=\frac{\alpha v^{3}}{e^{\frac{h v}{k T}}-1}
$$

We note here that the nonclassical assumption in Einstein derivation is the stationary energy states of the atom. ${ }^{9}$

It's worth mentioning here as we noted in the Planck's derivation that the elements of the canonical ensemble (which are atoms in our case) have to be able to exchange the quanta of energy between each other. This is done in our case by atoms emitting photons that are absorbed by other atoms. We note also that the atoms were assumed to have two level only while the fact that the spectrum of the blackbody radiation is continuous in frequency necessitates that all frequency resonances have to be present in the atoms of the wall and not only a discrete set of them. I think this problem is resolved by the presence of broadening mechanisms that leads eventually to the continuous spectrum. But shouldn't the different intensities of different lines affect the radiation spectrum of the blackbody?

\section{3-Third derivation using Bose-Einstein statistics}

A third derivation in which Pathria in his book attributed to Einstein, uses the Bose-Einstein statistics for the grand canonical ensemble. Here the radiation in the cavity is treated as a gas of indistinguishable photons obeying Bose statistics, namely the expectation number of the

photons occupying a certain energy level $\varepsilon$ is given by $\left\langle n_{\varepsilon}>=\frac{1}{e^{\varepsilon / k T}-1}\right.$. However several comments are in order here. First, the postulate of equal apriori probabilities implies that the system will be in the macrostate (e.g, E) that has the largest number of microstates. This is based on the assumption that at the same macrostate, the system can jump freely between different allowed microstates without restriction. For the case of ideal gas, these microstates may correspond to different energy distributions between the particles (or different space configurations). This of course requires a means of energy transfer between the particles which truly exists in the ideal gas through collisions. But what about the system of bosonic photons we have here? How can the system jump from one microstate to another and how 
can the photons exchange energy with one another? ${ }^{\mathrm{c}}$ As for the chemical potential, Pathria set it to zero. He said that because the number of photons is indefinite since photons are being absorbed and emitted from the walls continously, but isn't this the case for the grand canonical ensemble? Moreover I expect that at equilibrium the number of photons is fixed and not indefinite as Pathria stated, and hence we should use the canonical distribution rather than the grand canonical. However regardless of the type of the ensemble we use, the most probable value for $n_{\varepsilon}$ calculated without referring to a certain ensemble has the same value as $\left\langle n_{\varepsilon}\right\rangle$. But still I couldn't find a satisfactory explanation of setting the chemical potential of photons to zero. Next steps in this derivations are to calculate the density of states $g(\omega) d \omega$. In terms of the phase space $g(\omega) d \omega=2 \int \frac{d q^{3} 4 \pi p^{2} d p}{h^{3}}$ where the factor 2 is used to account for two perpendicular directions of polarizations of the photon spin. Using the relation between momentum and frequency $p=\frac{\hbar \omega}{c} \mathrm{we}$ find that $g(\omega) d \omega=2 \frac{V}{h^{3}}\left\{4 \pi\left(\frac{\hbar \omega}{c}\right)^{2}\left(\frac{\hbar d \omega}{c}\right)\right\}=\frac{V \omega^{2} d \omega}{\pi^{2} c^{3}}{ }^{\mathrm{d}}$ By expressing the energy level $\varepsilon$

as $\varepsilon=\hbar \omega$ we get exactly the same relation of the radiation density as that of Planck.

It's interesting that the assumption of the quantized nature of radiation inside the cavity (that light waves are made up of particles called photons) is sufficient to impose the quantized energy levels on atoms in equilibrium with light.

\section{4- Poincaré's last memoir on the quantum discontinuity of nature}

Here I'm going to comment on another proof of the quantum nature of light initiated by the famous French mathematician and physicist Henri Poincare. Since Poincare spent most of his life as a certified master of classical physics one would expect that he would be very reluctant at the age of 57 to change his mind about the nature of continuity of matter and radiation. However upon attending the Solvay conference that was held specially to investigate the new ideas of the quantum nature of light and to shed more light on the blackbody radiation, the "most mysterious phenomenon and a most difficult one to unveil" he was very enthusiastic upon the new idea and actively participated on the discussions. After the conference, Poincare returned to Paris and became engaged with the quantum problem. In accord to my objection to the third proof given above, Poincare considered Planck's proof lacking a very important component, the mechanism to achieve equilibrium between in the system of harmonic oscillators. In his own words, Poincare wrote: "For a 
(equilibrium) distribution to take place between the resonators of different wavelengths whose oscillations are the cause of radiation, the resonators must be capable of exchanging their energy. Otherwise, the initial distribution will persist indefinitely and this initial distribution is arbitrary, there could be no unique law of radiation."

However, I don't agree with that since Planck had to assume only that harmonic oscillators of the same frequency are in equilibrium. . In this case energy can be transferred between them without any problem since they are in resonance with one another There is no need to find a mechanism for exchanging energy between oscillators of different frequencies. To provide this mechanism, Poincare modeled the interaction between harmonic oscillators as mediated by a medium of freely moving atoms which collide with the resonators.

\section{5- Quantum statistics of photons}

The quantum first order degree of coherence which identifies the degree of correlation between the light fields at two space time points $\left(\mathrm{r}_{1}, \mathrm{t}_{1}\right)$ and $\left(\mathrm{r}_{2}, \mathrm{t}_{2}\right)$ is defined by

$$
g^{(1)}\left(r_{1} t_{1}, r_{2} t_{2}\right)=\frac{\left\langle\hat{E}^{-}\left(r_{1} t_{1}\right) \hat{E}^{+}\left(r_{2} t_{2}\right)\right\rangle}{\left[\left\langle\hat{E}^{-}\left(r_{1} t_{1}\right) \hat{E}^{+}\left(r_{1} t_{1}\right)\right\rangle\left\langle\hat{E}^{-}\left(r_{2} t_{2}\right) \hat{E}^{+}\left(r_{2} t_{2}\right)\right\rangle\right]^{\frac{1}{2}}}
$$

Where $\hat{E}^{-}\left(r_{1} t_{1}\right), \hat{E}^{+}\left(r_{2} t_{2}\right)$ are the electric field operators. The subscripts designating a certain mode and polarization of the field have been omitted to simplify notation. A fuller representation of the electric field operator is given by

$\widehat{E}_{\vec{k}, \vec{\sigma}}(r, t)=\widehat{E}^{-} \vec{k}, \vec{\sigma}(r, t)+\widehat{E}^{+} \vec{k}, \vec{\sigma}(r, t)$

$=C\left[\hat{a}_{\vec{k}, \vec{\sigma}} \exp (-i \omega t+i \vec{k} \cdot \vec{r})-\hat{a}^{+} \vec{k}, \vec{\sigma} \exp (i \omega t-i \vec{k} \cdot \vec{r})\right]$

The expectation values are calculated using the density operator, $\left\langle\hat{E}^{-}\left(r_{1} t_{1}\right) \hat{E}^{+}\left(r_{1} t_{1}\right)\right\rangle=\operatorname{Tr}\left(\hat{\rho} \hat{E}^{-}\left(r_{1} t_{1}\right) \hat{E}^{+}\left(r_{1} t_{1}\right)\right)$

The quantity $g^{(1)}\left(r_{1} t_{1}, r_{2} t_{2}\right)$ signifies the ability of the light at the two space time points to form interference fringes when superimposed. By expanding the expressions of the field operator, it can be seen that the coherence function is a function of the parameter $\tau=t_{2}-t_{1}+\frac{\vec{r}_{1}-\vec{r}_{2}}{c}$, hence it can be written for short as $g^{(1)}(\tau)$. First order coherence has similar properties in classical and quantum representations of light. $g^{(1)}(\tau)$ has the same numerical range in both schemes, namely $1 \geq\left|g^{(1)}(\tau)\right| \geq 0$. The interference pattern which is a result of the first order coherence can be obtained using both treatments. However more striking differences occur in measurements that depend on second order coherence. ${ }^{3}$

In a similar way the quantum degree of second order coherence is defined as 
$g^{(2)}\left(r_{1} t_{1}, r_{2} t_{2} ; r_{2} t_{2,} r_{1} t_{1}\right)=\frac{\left\langle\hat{E}^{-}\left(r_{1} t_{1}\right) \hat{E}^{-}\left(r_{2} t_{2}\right) \hat{E}^{+}\left(r_{1} t_{1}\right) \hat{E}^{+}\left(r_{2} t_{2}\right)\right\rangle}{\left\langle\widehat{E}^{-}\left(r_{1} t_{1}\right) \hat{E}^{+}\left(r_{1} t_{1}\right)\right\rangle\left\langle\hat{E}^{-}\left(r_{2} t_{2}\right) \hat{E}^{+}\left(r_{2} t_{2}\right)\right\rangle}$

The second order coherence defines the correlation between the light intensities in two different space time points. With intensities measured by phototubes, the correlation is proportional to the transition rate of the joint absorption of photons at the two space time points. ${ }^{3}$ Note that the sequence of operators matters in this definition, i.e, annihilation operators to the left. The second order coherence can be measured in a photon beam at the same loction using a single photon detector by counting the numbers of photons registered by the detector in brief in two brief intervals separated by time $\tau$, then the second order coherence is given by the correlation between the photon numbers as $g^{(2)}(\tau)=\langle n(0) n(\tau)\rangle / \bar{n}^{2}$

\section{6-Thermal (chaotic) photon states}

Since photons are bosons, they follow Bose-Einstein statistics and the probability of occupying the mode of energy $\varepsilon$ is given by (See Pathria 6.4.10)

$P_{\varepsilon}(n)=\frac{\left(e^{-\beta \varepsilon}\right)^{n}}{\left(1-e^{-\beta \varepsilon}\right)}$, and since the mean number of photons $<\mathrm{n}>$ is given by $\langle n\rangle=\frac{1}{e^{\beta \varepsilon}-1}$, then

The probability to detect $\mathrm{n}$ photons in a small time $\tau, P(n)$ can be written as

$$
P(n)=\frac{\langle n\rangle^{n}}{(1+\langle n\rangle)^{n+1}}
$$

Hence, the density operator of a single mode of thermal (blackbody) radiation is described by

$$
\begin{aligned}
\hat{\rho} & =\frac{1}{Z} e^{-\beta \hat{H}}=\sum_{n=0}^{\infty} b_{n}|n\rangle\langle n|, \\
b_{n} & =\frac{\bar{n}^{n}}{(\bar{n}+1)^{n+1}}=\frac{1}{1+\bar{n}}\left(1+\frac{1}{\bar{n}}\right)^{-n},
\end{aligned}
$$

Where $|n\rangle=(n !)^{-\frac{1}{2}}\left(a^{+}\right)^{n}|0\rangle$ and $\bar{n}$ is the mean number of photons detected in a certain time

$(\tau)$ and is given by

$$
\langle\hat{N}\rangle=\operatorname{tr}(\hat{\rho} \hat{N})=\frac{1}{e^{\beta \hbar \omega}-1}=\bar{n},
$$

And $\mathrm{Z}$ is the partition function given by :

$$
Z=1 /(1-\exp (-\beta \hbar \omega))
$$

And $|n\rangle$ is the number state of a single mode 
The variance of the photon number in thermal states is given by

$$
(\Delta N)^{2}=\bar{n}(\bar{n}+1)
$$

\section{7-Photon statistics of a coherent source}

A coherent source emits photons in states of a coherent superposition of number states in the form

$$
|\alpha\rangle=e^{-\frac{1}{2}|\alpha|^{2}} \sum_{n=0}^{\infty} \frac{\alpha^{n}}{\sqrt{n !}}|n\rangle .
$$

Where $\alpha$ in general is a complex number. Laser beams are best characterized by these states since they have the minimum amount of uncertainty in the phase and amplitude. The mean number of photons in the coherent states $\bar{n}$ is given by the relation $\bar{n}=|\alpha|^{2}$ And the probability to detect $\mathrm{n}$ photons $\mathrm{P}(\mathrm{n})$ is

$$
p_{n}=|\langle n \mid \alpha\rangle|^{2}=e^{-\bar{n}} \frac{\bar{n}^{n}}{n !} \text {. }
$$

We notice that this is a Poissonian distribution. We remember that this distribution is characteristic of processes involving the occurrence of discrete events happening independent of each other. Thus, in a coherent state photons behave like they were uncorrelated classical objects! In contrast to naive expectations, the photons in a (single mode) laser (and well above the threshold) arrive in a random fashion; in particular they do not "ride" on the electrical field maxima. ${ }^{8}$ The counting statistics are the same as the statistics of telephone calls arriving at a switchboard or web pages requests from a web server.

As in possonian distribution, the variance of the number of detected photons $\Delta n$ is given by the relation $|\Delta n|^{2}=\bar{n}$. A coherent state of radiation is first order coherent, that is $\left|g^{(1)}(\tau)\right|=1$ for all pairs of space time points. A comparison between the photo-count distribution of the thermal (chaotic) light and the coherent light is illustrated in figure( )

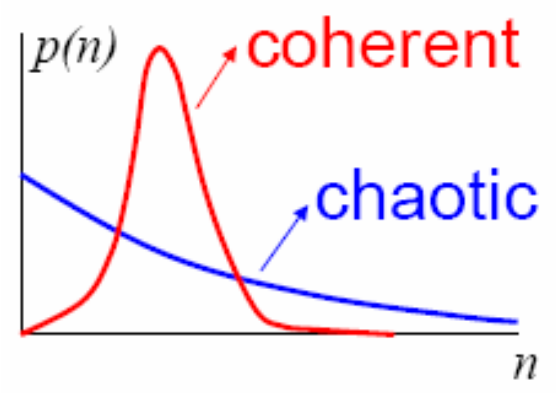


The photo-count distribution in this graph is obtained experimentally by letting the light fall on a photo-detector through a shutter that is open for a very short interval. And counting the number of photons registered by the photo-detector. This measurement is repeated many times and a time delay is allowed between successive measurements. This delay should be long compared to the coherent time of the light.

It's interesting to note that the photon count distribution of both coherent and chaotic light can also be obtained from other arguments as Loudon did in 6.6, 6.7 in his book. The only assumption made are that the number of photons detected by the photodetctor is proportional to the intensity of the light, and another assumption pertinent to the type of radiation. For coherent radiation the other assumption is that the intensity of light is independent of time, while for chaotic light the intensity has a Gaussian distribution and the averaging process over time is equivalent to the ensemble averging by the ergodicity theorem. The derivation is lengthy and can be reviewed from Loudon's book.

It's worth mentioning that thermal photon statistics can be obtained by a superposition of many coherent states in which a changes randomly in Gaussian distribution given by

$$
P(\alpha)=\frac{1}{\pi\left\langle\left|\alpha^{2}\right|\right\rangle} e^{\frac{-|\alpha|^{2}}{\left\langle\|\left.\alpha\right|^{2}\right\rangle}}
$$

And hence the density operator will be given by

$$
\rho_{\text {chaotic }}=\frac{1}{\pi\langle n\rangle} \int^{-\frac{|\alpha|^{2}}{\langle n\rangle}}|\alpha\rangle\langle\alpha| d^{2} \alpha
$$

Where $\langle n\rangle$ is given by

$$
\left\langle|\alpha|^{2}\right\rangle_{A V .}=\langle n\rangle
$$

A comparison between the distribution of $\alpha$ in the coherent (laser) source and the thermal source is shown in figure()

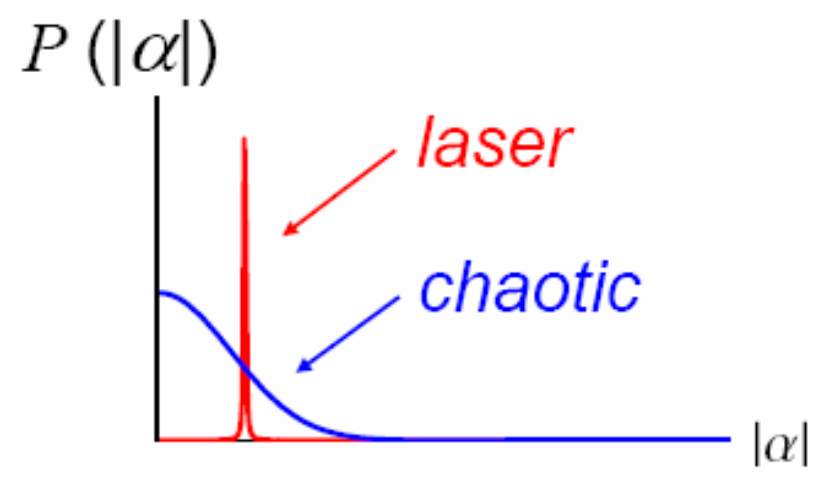

Another important aspect in the comparison between chaotic light and laser is the bunching effect. 
Photon bunching is the tendency of photons to group together in bunches, if the photodetctor detected $\mathrm{n}$ photons, it's more probable that these $\mathrm{n}$ photons reached the photodetector close to each other than spread in time.

As shown in figure ( ), chaotic light has strong correlation $g^{(2)}(\tau)$ at shorter times that decreases as $\tau$ increases, while the photons of the laser beam arrive at the photodetector uncorrelated-as mentioned before- an exhibits no bunching.

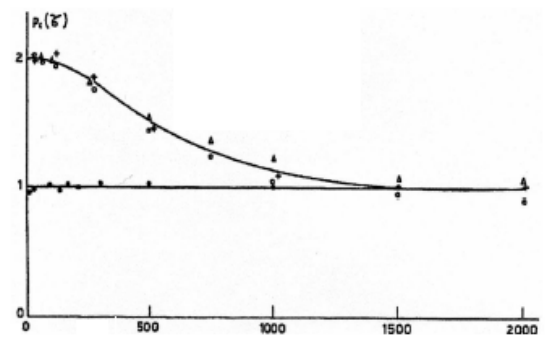

\section{8- Non-classical properties of light}

Now we turn our attention to two other distributions of light that are manifestations of certain nonclassical states of light, namely photon anti-bunching and sub-poissonian photon statistics.

Anti-bunching is the opposite effect i.e., the photons prefer to come not too close. In a quantitative manner, photon anti-bunching occurs of the second order coherence increases from its initial value at $\tau=0{ }^{11}$ or equivalently

$g^{(2)}(t, t+\tau)>g^{(2)}(t, t)$

Another criterion for photon anti-bunching is $1>g^{(2)}(0) \geq 0$

Histogram of time delays between consecutive photon pairs in photon anti-bunched beam is shown in figure() 


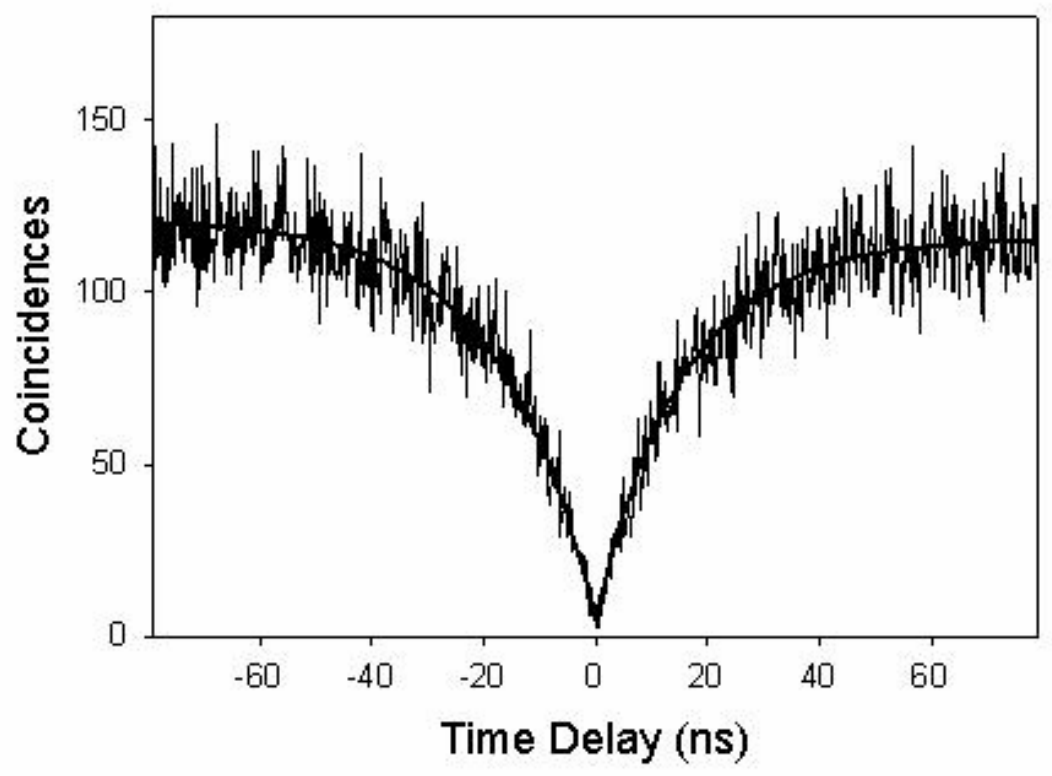

The phenomenon of anti-bunching or anti-correlation is used as an evidence for the quantum nature of radiation in some experiments. ${ }^{12}$

Unlike Poissonian photon statistics, sub-Poisson photon statistics is a photon number distribution for which the variance is less than the mean and super-Poisson statistics is a photon number distribution for which the variance is greater than the mean. Both photon count statistics compared to Poisson statistics are shown in the figure()

It has been shown by Mandel and Zou ${ }^{10}$ that these two nonclassical phenomena are not related and needn't occur together as it was thought previously by some authors.

\section{9-Conclusion}

The quantization of the electromagnetic energy, had the profound impact to quantize many other quantities.

\section{0-References}

1- Max Planck: the reluctant revolutionary, Helge Kragh, Physics World, December 2000.

2- 100 Years of Light Quanta, Roy J. Glauber, Nobel Lecture

3- The Quantum Theory of Light, 2nd edition, Roudney Loudon.

4- On the Law of Distribution of Energy in the Normal Spectrum, Max Planck Annalen der Physik vol. 4, p. 553 ff (1901)

5- The evolution of radiation toward thermal equilibrium: A soluble model that illustrates the foundations of statistical mechanics, Michael Nauenberg, Am. J. Phys. $72 \sim 3$ !, March 2004 
6- Statistical Mechanics, 2nd edition, Pathria

7- Poincaré's proof of the quantum discontinuity of nature Jeffrey J. Prentis Am. J. Phys. 63, 339 (1995)

8- Photons and Photon Statistics: From Incandescent Light to Lasers, Ralph v. Baltz

9- Einstein's derivation of Planck's radiation law, Henry R. Lewis, Am. J. Phys. 41, January 1973

10- Photon-antibunching and sub-Poissonian photon statistics, X. T. Zou and L. Mandel, Phys. Rev. A 41, 475 - 476

11-Various Approaches to photon AntiBunching in Second Harmonic generation, A. Miranowicz et.al

12- In an experiment by a group in L'ECOLE NORMALE SUPERIEURE DE CACHAN, a light beam is let to pass through a biprism. The two beams, leaving the prism are detected using an avalanche photodetctor and the output of the light beams are correlated (i.e, the number of coincednces is calculated). "If light is really made of quanta, a single photon should either be deviated upwards or downwards, but should not be split by the biprism. In that case, no coincidences corresponding to joint photodetections on the two output beams should be observed. On the opposite, for a semiclassical model that describes light as a classical wave, the input wavefront will be split in two equal parts, leading to a non-zero probability of joint detection on the two photodetectors. Observation of zero coincidences, corresponding to an anticorrelation effet, would thus give evidence for a particle-like behaviour." See http://www.physique.ens-cachan.fr/franges_photon/anticorrelation.htm

13-http://www.stanford.edu/group/moerner/QD.html 